\title{
Planar Decompositions and the Crossing Number of Graphs with an Excluded Minor*
}

\author{
David R. Wood ${ }^{1, \star \star}$ and Jan Arne Telle ${ }^{2}$ \\ ${ }^{1}$ Departament de Matemática Aplicada II, Universitat Politècnica de Catalunya, \\ Barcelona, Spain \\ david. wood@upc . edu \\ ${ }^{2}$ Department of Informatics, The University of Bergen, Bergen, Norway \\ Jan.Arne.Telle@ii.uib.no
}

\begin{abstract}
Tree decompositions of graphs are of fundamental importance in structural and algorithmic graph theory. Planar decompositions generalise tree decompositions by allowing an arbitrary planar graph to index the decomposition. We prove that every graph that excludes a fixed graph as a minor has a planar decomposition with bounded width and a linear number of bags.

The crossing number of a graph is the minimum number of crossings in a drawing of the graph in the plane. We prove that planar decompositions are intimately related to the crossing number, in the sense that a graph with bounded degree has linear crossing number if and only if it has a planar decomposition with bounded width and linear order. It follows from the above result about planar decompositions that every graph with bounded degree and an excluded minor has linear crossing number.

Analogous results are proved for the convex and rectilinear crossing numbers. In particular, every graph with bounded degree and bounded tree-width has linear convex crossing number, and every $K_{3,3}$-minor-free graph with bounded degree has linear rectilinear crossing number.
\end{abstract}

\section{Introduction}

The crossing number of a graph $G$, denoted by $\operatorname{cr}(G)$, is the minimum number of crossings in a drawing 1 of $G$ in the plane; see the survey [16]. Crossing number is an important measure of non-planarity, with applications in discrete and computational geometry, graph visualisation, and VLSI circuit design.

* The full version of this extended abstract is reference [17].

** The research of David Wood is supported by a Marie Curie Fellowship of the European Community under contract 023865, and by the projects MCYT-FEDER BFM2003-00368 and Gen. Cat 2001SGR00224.

${ }^{1}$ A drawing of a graph represents each vertex by a distinct point in the plane, and represents each edge by a simple closed curve between its endpoints, such that the only vertices an edge intersects are its own endpoints, and no three edges intersect at a common point (except at a common endpoint). A crossing is a point of intersection between two edges (other than a common endpoint). Undefined terminology can be found in the monographs $[5,10]$. 
Upper bounds on the crossing number are the focus of this paper. Obviously $\operatorname{cr}(G) \leq\left(\begin{array}{c}\|G\| \\ 2\end{array}\right)$ for every graph $G$, where $|G|:=|V(G)|$ and $\|G\|:=|E(G)|$. A graph family $\mathcal{F}$ has linear crossing number if for some constant $c$, every graph $G \in \mathcal{F}$ has crossing number $\operatorname{cr}(G) \leq c|G|$. For example, Pach and Tóth [1] proved that graphs of bounded genus and bounded degree have linear crossing number. Our main result states that bounded-degree graphs that exclude a fixed graph as a minor have linear crossing number.

Theorem 1. For every graph $H$ there is a constant $c=c(H)$, such that every $H$-minor-free graph $G$ has crossing number at most $c \Delta(G)^{2}|G|$.

Theorem 1 implies the above-mentioned result of Pach and Tóth [11], since graphs of bounded genus exclude a fixed graph as a minor (although the dependence on $\Delta$ is different in the two proofs; see Section 5). Moreover, there are graphs with a fixed excluded minor and unbounded genus. For other recent work on minors and crossing number see [3, 8].

Note that the assumption of bounded degree in Theorem 1 is unavoidable. For example, the complete bipartite graph $K_{3, n}$ has no $K_{5}$-minor, yet has $\Omega\left(n^{2}\right)$ crossing number [12]. Conversely, bounded degree does not by itself guarantee linear crossing number. For example, a random cubic graph on $n$ vertices has $\Omega(n)$ bisection width, which implies that it has $\Omega\left(n^{2}\right)$ crossing number. Also note that $c \leq \frac{20}{3}$ in Theorem 1 with $H=K_{5}$; see [17]. The proof of Theorem 1 is based on planar decompositions, which are introduced in the next section.

\section{Graph Decompositions}

Let $G$ and $D$ be graphs, such that each vertex of $D$ is a set of vertices of $G$ (called a $b a g$ ). We allow distinct vertices of $D$ to be the same set of vertices in $G$; that is, $V(D)$ is a multiset. For each vertex $v$ of $G$, let $D(v)$ be the subgraph of $D$ induced by the bags that contain $v$. Then $D$ is a decomposition 2 of $G$ if:

- $D(v)$ is connected and nonempty for each vertex $v$ of $G$, and

- $D(v)$ and $D(w)$ touch 3 for each edge $v w$ of $G$.

Let $D$ be a decomposition of a graph $G$. The width of $D$ is the maximum cardinality of a bag. The order of $D$ is the number of bags. $D$ has linear order if its order is $\mathcal{O}(|G|)$. If the graph $D$ is a tree, then the decomposition $D$ is a tree decomposition. If the graph $D$ is a cycle, then the decomposition $D$ is a cycle decomposition. The decomposition $D$ is planar if the graph $D$ is planar. The genus of the decomposition $D$ is the genus of the graph $D$.

A decomposition $D$ of a graph $G$ is strong if $D(v)$ and $D(w)$ intersect for each edge $v w$ of $G$. The tree-width of $G$, denoted by $\operatorname{tw}(G)$, is 1 less than the minimum

${ }^{2}$ Decompositions, when $D$ is a tree, were introduced by Robertson and Seymour. Diestel and Kühn [6] first generalised the definition for arbitrary graphs $D$.

${ }^{3}$ Subgraphs $A$ and $B$ of a graph $G$ intersect if $V(A) \cap V(B) \neq \emptyset$, and $A$ and $B$ touch if they intersect or $v \in V(A)$ and $w \in V(B)$ for some edge $v w$ of $G$. 
width of a strong tree decomposition of $G$. For example, a graph has tree-width 1 if and only if it is a forest. Graphs with tree-width 2 (called series-parallel) are planar, and are characterised as those graphs with no $K_{4}$-minor. Tree-width is particularly important in structural and algorithmic graph theory.

For applications to crossing number, tree decompositions are not powerful enough: even the $n \times n$ planar grid has tree-width $n$. Lemmas 10] and 11 in Section 4 prove the following theorem, which says that planar decompositions are the right type of decomposition for applications to crossing number.

Theorem 2. A family of graphs with bounded degree has linear crossing number if and only if every graph in the family has a planar decomposition with bounded width and linear order.

Every tree $T$ satisfies the Helly property: every collection of pairwise intersecting subtrees of $T$ have a vertex in common. It follows that if a tree $T$ is a strong decomposition of $G$ then every clique of $G$ is contained in some bag of $T$. Other graphs do not have this property. It will be desirable (for performing $k$-sums in Section 3) that (non-tree) decompositions have a similar property. We therefore introduce the following definitions.

For $p \geq 0$, a $p$-clique is a clique of cardinality $p$. A $(\leq p)$-clique is a clique of cardinality at most $p$. For $p \geq 2$, a decomposition $D$ of a graph $G$ is a $p$ decomposition if each $(\leq p)$-clique of $G$ is a subset of some bag of $D$, or is a subset of the union of two adjacent bags of $D$. An $\omega(G)$-decomposition of $G$ is called an $\omega$-decomposition, where $\omega(G)$ is the maximum cardinality of a clique of $G$. A $p$-decomposition $D$ of $G$ is strong if each $(\leq p)$-clique of $G$ is a subset of some bag of $D$. Observe that a (strong) 2-decomposition is the same as a (strong) decomposition, and a (strong) $p$-decomposition also is a (strong) $q$-decomposition for all $q \in[2, p]$.

In Section 6, we prove the following theorem, which is one of the main contributions of the paper.

Theorem 3. For every graph $H$ there is an integer $k=k(H)$, such that every $H$-minor-free graph $G$ has a planar $\omega$-decomposition of width $k$ and order $|G|$.

\section{Manipulating Decompositions}

In this section we describe four tools for manipulating graph decompositions. Our first tool describes the effect of contracting an edge in a decomposition. The elementary proof is in the full paper.

Lemma 4 ([17]). Suppose that $D$ is a planar (strong) p-decomposition of a graph $G$ with width $k$. Say $X Y$ is an edge of $D$. Then the decomposition $D^{\prime}$ obtained by contracting the edge $X Y$ into the vertex $X \cup Y$ is a planar (strong) p-decomposition of $G$ with width $\max \{k,|X \cup Y|\}$. In particular, if $|X \cup Y| \leq k$ then $D^{\prime}$ also has width $k$. 
Lemma 5. Suppose that a graph $G$ has a (strong) planar p-decomposition $D$ of width $k$ and order at most $c|G|$ for some $c \geq 1$. Then $G$ has a (strong) planar p-decomposition of width $c^{\prime} k$ and order $|G|$, for some $c^{\prime}$ depending only on $c$.

Proof. Without loss of generality, $D$ is a planar triangulation. By a result of Biedl et al. [1], $D$ has a matching $M$ of at least $\frac{1}{3}|D|$ edges. Applying Lemma 4 to each edge of $M$, we obtain a (strong) planar $p$-decomposition of $G$ with width at most $2 k$ and order at most $\frac{2}{3}|D|$. By induction, for every integer $i \geq 1, G$ has a (strong) planar $p$-decomposition of width $2^{i} k$ and order at most $\left(\frac{2}{3}\right)^{i}|D|$. With $i:=\left\lceil\log _{3 / 2} c\right\rceil$, the assumption that $|D|=c|G|$ implies that $G$ has a (strong) planar $p$-decomposition of width $2^{i} k$ and order $|G|$.

Our second tool describes how two decompositions can be composed.

Lemma 6. Suppose that $D$ is a (strong) p-decomposition of a graph $G$ with width $k$, and that $J$ is a decomposition of $D$ with width $\ell$. Then $G$ has a (strong) p-decomposition isomorphic to $J$ with width $k \ell$.

Proof. Let $J^{\prime}$ be the graph isomorphic to $J$ that is obtained by renaming each bag $Y \in V(J)$ by $Y^{\prime}:=\{v \in V(G): v \in X \in Y$ for some $X \in V(D)\}$. There are at most $\ell$ vertices $X \in Y$, and at most $k$ vertices $v \in X$. Thus each bag of $J^{\prime}$ has at most $k \ell$ vertices. First we prove that $J^{\prime}(v)$ is connected for each vertex $v$ of $G$. Let $A^{\prime}$ and $B^{\prime}$ be two bags of $J^{\prime}$ that contain $v$. Let $A$ and $B$ be the corresponding bags in $D$. Thus $v \in X_{1}$ and $v \in X_{t}$ for some bags $X_{1}, X_{t} \in V(D)$ such that $X_{1} \in A$ and $X_{t} \in B$ (by the construction of $J^{\prime}$ ). Since $D(v)$ is connected, there is a path $X_{1}, X_{2}, \ldots, X_{t}$ in $D$ such that $v$ is in each $X_{i}$. In particular, each $X_{i} X_{i+1}$ is an edge of $D$. Now $J\left(X_{i}\right)$ and $J\left(X_{i+1}\right)$ touch in $J$. Thus there is path in $J$ between any vertex of $J$ that contains $X_{1}$ and any vertex of $J$ that contains $X_{t}$, such that every bag in the path contains some $X_{i}$. In particular, there is a path $P$ in $J$ between $A$ and $B$ such that every bag in $P$ contains some $X_{i}$. Let $P^{\prime}:=\left\{Y^{\prime}: Y \in P\right\}$. Then $v \in Y^{\prime}$ for each bag $Y^{\prime}$ of $P^{\prime}$ (by the construction of $J^{\prime}$ ). Thus $P^{\prime}$ is a connected subgraph of $J^{\prime}$ that includes $A^{\prime}$ and $B^{\prime}$, and $v$ is in every such bag. Therefore $J^{\prime}(v)$ is connected. In the full paper [17] we prove that for each $(\leq p)$-clique $C$ of $G$, (a) $C$ is a subset of some bag of $J^{\prime}$, or (b) $C$ is a subset of the union of two adjacent bags of $J^{\prime}$.

Moreover, if $D$ is strong then case (a) always occurs.

The third tool converts a decomposition into an $\omega$-decomposition with a small increase in the width. A graph $G$ is $d$-degenerate if every subgraph of $G$ has a vertex of degree at most $d$.

Lemma 7. Every d-degenerate graph $G$ has a strong $\omega$-decomposition isomorphic to $G$ of width at most $d+1$.

Proof. It is well known (and easily proved) that $G$ has an acyclic orientation such that each vertex has indegree at most $d$. Replace each vertex $v$ by the bag $\{v\} \cup$ $N_{G}^{-}(v)$. Every subgraph of $G$ has a sink. Thus every clique is a subset of some bag. The set of bags that contain a vertex $v$ are indexed by $\{v\} \cup N_{G}^{+}(v)$, which 
induces a connected subgraph in $G$. Thus we have a strong $\omega$-decomposition. Each bag has cardinality at most $d+1$.

Lemmas 6 and 7 imply:

Lemma 8. Suppose that $D$ is a decomposition of a d-degenerate graph $G$ of width $k$. Then $G$ has a strong $\omega$-decomposition isomorphic to $D$ of width $k(d+1)$.

Our fourth tool describes how to determine a planar decomposition of a cliquesum of two graphs, given planar decompositions of the summand: 4 . Let $G_{1}$ and $G_{2}$ be disjoint graphs. Suppose that $C_{1}$ and $C_{2}$ are $k$-cliques of $G_{1}$ and $G_{2}$ respectively, for some integer $k \geq 0$. Let $C_{1}=\left\{v_{1}, v_{2}, \ldots, v_{k}\right\}$ and $C_{2}=$ $\left\{w_{1}, w_{2}, \ldots, w_{k}\right\}$. Let $G$ be a graph obtained from $G_{1} \cup G_{2}$ by identifying $v_{i}$ and $w_{i}$ for each $i \in[1, k]$, and deleting an arbitrary (possibly empty) subset of the edges between vertices in $C_{1}\left(=C_{2}\right)$. Then $G$ is a $k$-sum of $G_{1}$ and $G_{2}$. An $\ell$-sum for some $\ell \leq k$ is called a $(\leq k)$-sum. For example, if $G_{1}$ and $G_{2}$ are planar then it is easily seen that every $(\leq 2)$-sum of $G_{1}$ and $G_{2}$ is also planar.

Lemma 9. Suppose that for integers $p \leq q$, a graph $G$ is a $(\leq p)$-sum of graphs $G_{1}$ and $G_{2}$, and each $G_{i}$ has a (strong) planar q-decomposition $D_{i}$ of width $k_{i}$. Then $G$ has a (strong) planar $q$-decomposition of width $\max \left\{k_{1}, k_{2}\right\}$ and order $\left|D_{1}\right|+\left|D_{2}\right|$.

Proof. Let $C:=V\left(G_{1}\right) \cap V\left(G_{2}\right)$. Then $C$ is a $(\leq p)$-clique, and thus a $(\leq q)$ clique, of both $G_{1}$ and $G_{2}$. Thus for each $i,(1) C \subseteq X_{i}$ for some bag $X_{i}$ of $D_{i}$, or (2) $C \subseteq X_{i} \cup Y_{i}$ for some edge $X_{i} Y_{i}$ of $D_{i}$. If (1) is applicable, which is the case if $D_{i}$ is strong, then consider $Y_{i}:=X_{i}$ in what follows.

Let $D$ be the graph obtained from the disjoint union of $D_{1}$ and $D_{2}$ by adding edges $X_{1} X_{2}, X_{1} Y_{2}, Y_{1} X_{2}$, and $Y_{1} Y_{2}$. By considering $X_{1} Y_{1}$ to be on the outerface of $G_{1}$ and $X_{2} Y_{2}$ to be on the outerface of $G_{2}$, observe that $D$ is planar, as illustrated in Figure 1.

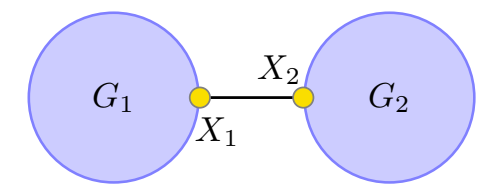

(a)

Fig. 1. Sum of (a) strong planar decompositions, (b) planar decompositions

We now prove that $D(v)$ is connected for each vertex $v$ of $G$. If $v \notin V\left(G_{1}\right)$ then $D(v)=D_{2}(v)$, which is connected. If $v \notin V\left(G_{2}\right)$ then $D(v)=D_{1}(v)$, which

\footnotetext{
${ }^{4}$ Leaños and Salazar [9] recently proved related results on the additivity of crossing numbers.
} 
is connected. Otherwise, $v \in C$. Thus $D(v)=D_{1}(v) \cup D_{2}(v)$. Since $v \in X_{1} \cup Y_{1}$ and $v \in X_{2} \cup Y_{2}$, and $X_{1}, Y_{1}, X_{2}, Y_{2}$ induce a connected subgraph $\left(\subseteq K_{4}\right)$ in $D$, we have that $D(v)$ is connected.

Each $(\leq q)$-clique $B$ of $G$ is a $(\leq q)$-clique of $G_{1}$ or $G_{2}$. Thus $B$ is a subset of some bag of $D$, or $B$ is a subset of the union of two adjacent bags of $D$. Moreover, if $D_{1}$ and $D_{2}$ are both strong, then $B$ is a subset of some bag of $D$. Therefore $D$ is a $q$-decomposition of $G$, and if $D_{1}$ and $D_{2}$ are both strong then $D$ is also strong. The width and order of $D$ are obviously as claimed.

\section{Planar Decompositions and the Crossing Number}

The following lemma is the key link between planar decompositions and the crossing number of a graph.

Lemma 10. Suppose that $D$ is a planar decomposition of a graph $G$ of width $k$. Then the crossing number of $G$ satisfies

$$
\operatorname{cr}(G) \leq 2 \Delta(G)^{2} \sum_{X \in V(D)}\left(\begin{array}{c}
|X|+1 \\
2
\end{array}\right) \leq k(k+1) \Delta(G)^{2}|D| .
$$

Proof. Fix a straight-line drawing of $D$ with no crossings. Let $\epsilon>0$. Let $R_{\epsilon}(X)$ be the open disc of radius $\epsilon$ centred at each vertex $X$ in the drawing of $D$. For each edge $X Y$ of $D$, let $R_{\epsilon}(X Y)$ be the union of all segments with one endpoint in $R_{\epsilon}(X)$ and one endpoint in $R_{\epsilon}(Y)$. For some $\epsilon>0, R_{\epsilon}(X) \cap R_{\epsilon}(Y)=\emptyset$ for all distinct bags $X$ and $Y$ of $D$, and $R_{\epsilon}(X Y) \cap R_{\epsilon}(A B)=\emptyset$ for all edges $X Y$ and $A B$ of $D$ that have no endpoint in common.

For each vertex $v$ of $G$, choose a bag $S_{v}$ of $D$ that contains $v$. For each vertex $v$ of $G$, choose a point $p(v) \in R_{\epsilon}\left(S_{v}\right)$, and for each bag $X$ of $D$, choose a set $P(X)$ of $\sum_{v \in X} \operatorname{deg}_{G}(v)$ points in $R_{\epsilon}(X)$, so that no two points coincide, no three points are collinear, and no three segments, each connecting two points, cross at a common point. These points can be chosen iteratively since each disc $R_{\epsilon}(X)$ is 2-dimensiona 5 , but the set of excluded points is 1-dimensional.

Draw each vertex $v$ at $p(v)$. For each edge $v w$ of $G$, a simple polyline $L(v w)=$ $\left(p(v), x_{1}, x_{2}, \ldots, x_{a}, y_{1}, y_{2}, \ldots, y_{b}, p(w)\right)$, defined by its endpoints and bends, is a feasible representation of $v w$ if:

(1) each bend $x_{i}$ is in $P\left(X_{i}\right)$ for some bag $X_{i}$ containing $v$,

(2) each bend $y_{i}$ is in $P\left(Y_{i}\right)$ for some bag $Y_{i}$ containing $w$,

(3) the bags $S_{v}, X_{1}, X_{2}, \ldots, X_{a}, Y_{1}, Y_{2}, \ldots, Y_{b}, S_{w}$ are distinct

(unless $S_{v}=S_{w}$ in which case $a=b=0$ ), and

(4) consecutive bends in $L(v w)$ occur in adjacent bags of $D$.

Since $D(v)$ and $D(w)$ touch, there is a feasible polyline that represents $v w$.

\footnotetext{
${ }^{5}$ Let $Q$ be a nonempty set of points in the plane. Then $Q$ is 2-dimensional if it contains a disk of positive radius; $Q$ is 1-dimensional if it is not 2-dimensional but contains a finite curve; otherwise $Q$ is 0 -dimensional.
} 
A drawing of $G$ is feasible if every edge of $G$ is represented by a feasible polyline, and no two bends coincide. Since each $|P(X)|=\sum_{v \in X} \operatorname{deg}(v)$, there is a feasible drawing. In particular, no edge passes through a vertex and no three edges have a common crossing point.

By properties (1)-(4), each segment in a feasible drawing is contained within $R_{\epsilon}(X)$ for some bag $X$ of $D$, or within $R_{\epsilon}(X Y)$ for some edge $X Y$ of $D$. Consider a crossing in $G$ between edges $v w$ and $x y$. Since $D$ is drawn without crossings, the crossing point is contained within $R_{\epsilon}(X)$ for some bag $X$ of $D$, or within $R_{\epsilon}(X Y)$ for some edge $X Y$ of $D$. Thus some endpoint of $v w$, say $v$, and some endpoint of $x y$, say $x$, are in a common bag $X$. In this case, charge the crossing to the 5-tuple $(v w, v, x y, x, X)$.

At most four crossings are charged to each 5-tuple $(v w, v, x y, x, X)$, since by property (4), each of $v w$ and $x y$ have at most two segments that intersect $R_{\epsilon}(X)$ (which might pairwise cross). We prove in [17] that in a feasible drawing that minimises the total (Euclidean) length of the edges (with $\{p(v): v \in V(G)\}$ and $\{P(X): X \in V(D)\}$ fixed), at most two crossings are charged to each such 5-tuple. Thus the number of crossings is at most twice the number of 5 -tuples. Therefore the number of crossings is at most

$$
2 \sum_{X \in V(D)} \sum_{v, x \in X} \operatorname{deg}_{G}(v) \cdot \operatorname{deg}_{G}(x) \leq 2 \Delta(G)^{2} \sum_{X \in V(D)}\left(\begin{array}{c}
|X|+1 \\
2
\end{array}\right) .
$$

Note that the bound on the crossing number in Lemma 10 is within a constant factor of optimal for the complete graph [17]. The following converse result to Lemma 10 is proved by replacing each crossing by a bag.

Lemma 11 ([17]). Every graph $G$ has a planar decomposition of width 2 and order $|G|+\operatorname{cr}(G)$.

\section{Graphs Embedded in a Surface}

Let $\mathbb{S}_{\gamma}$ be the orientable surface with $\gamma \geq 0$ handles. A cycle in $\mathbb{S}_{\gamma}$ is a closed curve in the surface. A cycle is contractible if it is contractible to a point in the surface. A noncontractible cycle is separating if it separates $\mathbb{S}_{\gamma}$ into two connected components.

The (orientable) genus of a graph $G$ is the minimum $\gamma$ such that $G$ has a 2-cell embedding in $\mathbb{S}_{\gamma}$. Let $G$ be a graph embedded in $\mathbb{S}_{\gamma}$. In what follows, by a face we mean the set of vertices on the boundary of the face. Let $F(G)$ be the set of faces in $G$. A noose of $G$ is a cycle $C$ in $\mathbb{S}_{\gamma}$ that does not intersect the interior of an edge of $G$. Let $V(C)$ be the set of vertices of $G$ intersected by $C$. The length of $C$ is $|V(C)|$.

Pach and Tóth [11] proved that, for some constant $c_{\gamma}$, the crossing number of every graph $G$ of genus $\gamma$ satisfies

$$
\operatorname{cr}(G) \leq c_{\gamma} \sum_{v \in V(G)} \operatorname{deg}(v)^{2} \leq 2 c_{\gamma} \Delta(G)\|G\|
$$


The constant $c_{\gamma}$ was subsequently improved by Djidjev and Vrťo [7]. It is well known [17] that $\|G\| \leq(\sqrt{3 \gamma}+3)|G|-6$. Thus

$$
\operatorname{cr}(G) \leq c_{\gamma} \Delta(G)|G|
$$

By Lemma 11, $G$ has a planar decomposition of width 2 and order $c_{\gamma} \Delta(G)|G|$. We now provide an analogous result without the dependence on $\Delta(G)$, but at the expense of an increased bound on the width.

Theorem 12. Every graph $G$ with genus $\gamma$ has a planar decomposition of width $2^{\gamma}$ and order $3^{\gamma}|G|$.

The key to the proof of Theorem 12 is the following lemma, whose proof is inspired by similar ideas of Pach and Tóth [11].

Lemma 13. Let $G$ be a graph with a 2-cell embedding in $\mathbb{S}_{\gamma}$ for some $\gamma \geq 1$. Then $G$ has a decomposition of width 2 , genus at most $\gamma-1$, and order $3|G|$.

Proof. Since $\gamma \geq 1, G$ has a noncontractible nonseparating noose. Let $C$ be a noncontractible nonseparating noose of minimum length $k:=|V(C)|$. Orient $C$ and let $V(C):=\left(v_{1}, v_{2}, \ldots, v_{k}\right)$ in the order around $C$. For each vertex $v_{i} \in$ $V(C)$, let $E^{\ell}\left(v_{i}\right)$ and $E^{r}\left(v_{i}\right)$ respectively be the set of edges incident to $v_{i}$ that are on the left-hand side and right-hand side of $C$ (with respect to the orientation). Cut the surface along $C$, and attach a disk to each side of the cut. Replace each vertex $v_{i} \in V(C)$ by two vertices $v_{i}^{\ell}$ and $v_{i}^{r}$ respectively incident to the edges in $E^{\ell}\left(v_{i}\right)$ and $E^{r}\left(v_{i}\right)$. Embed $v_{i}^{\ell}$ on the left-hand side of the cut, and embed $v^{r}$ on the right-hand side of the cut. We obtain a graph $G^{\prime}$ embedded in a surface of genus at most $\gamma-1$ (since $C$ is nonseparating).

Let $L:=\left\{v_{i}^{\ell}: v \in V(C)\right\}$ and $R:=\left\{v_{i}^{r}: v \in V(C)\right\}$. By Menger's Theorem, the maximum number of disjoint paths between $L$ and $R$ in $G^{\prime}$ equals the minimum number of vertices that separate $L$ from $R$ in $G^{\prime}$. Let $Q$ be a minimum set of vertices that separate $L$ from $R$ in $G^{\prime}$. Then there is a noncontractible nonseparating noose in $G$ that only intersects vertices in $Q$. (It is nonseparating in $G$ since $L$ and $R$ are identified in $G$.) Thus $|Q| \geq k$ by the minimality of $|V(C)|$. Hence there exist $k$ disjoint paths $P_{1}, P_{2}, \ldots, P_{k}$ between $L$ and $R$ in $G^{\prime}$, where the endpoints of $P_{i}$ are $v_{i}^{\ell}$ and $v_{\sigma(i)}^{r}$, for some permutation $\sigma$ of $[1, k]$. In the disc with $R$ on its boundary, draw an edge from each vertex $v_{\sigma(i)}^{r}$ to $v_{i}^{r}$ such that no three edges cross at a single point and every pair of edge cross at most once. Add a new vertex $x_{i, j}$ on each crossing point between edges $v_{\sigma(i)}^{r} v_{i}^{r}$ and $v_{\sigma(j)}^{r} v_{j}^{r}$. Let $G^{\prime \prime}$ be the graph obtained. Then $G^{\prime \prime}$ is embedded in $S_{\gamma-1}$.

We now make $G^{\prime \prime}$ a decomposition of $G$. Replace $v_{i}^{\ell}$ by $\left\{v_{i}\right\}$ and replace $v_{i}^{r}$ by $\left\{v_{i}\right\}$. Replace every other vertex $v$ of $G$ by $\{v\}$. Replace each 'crossing' vertex $x_{i, j}$ by $\left\{v_{i}, v_{j}\right\}$. Now for each vertex $v_{i} \in V(C)$, add $v_{i}$ to each bag on the path $P_{i}$ from $v_{i}^{\ell}$ to $v_{\sigma(i)}^{r}$. Thus $G^{\prime \prime}\left(v_{i}\right)$ is a (connected) path. Clearly $G^{\prime \prime}(v)$ and $G^{\prime \prime}(w)$ touch for each edge $v w$ of $G$. Hence $G^{\prime \prime}$ is a decomposition of $G$ with genus at most $\gamma-1$. Since the paths $P_{1}, P_{2}, \ldots, P_{k}$ are pairwise disjoint, the width of the decomposition is 2 . 
It remains to bound the order of $G^{\prime \prime}$. Let $n:=|G|$. Observe that $G^{\prime \prime}$ has at most $n+k+\left(\begin{array}{l}k \\ 2\end{array}\right)$ vertices. One of the paths $P_{i}$ has at most $\frac{n+k}{k}$ vertices. For ease of counting, add a cycle to $G^{\prime}$ around $R$. Consider the path in $G^{\prime}$ that starts at $v_{i}^{\ell}$, passes through each vertex in $P_{i}$, and then takes the shortest route from $v_{\sigma(i)}^{r}$ around $R$ back to $v_{i}^{r}$. The distance between $v_{\sigma(i)}^{r}$ and $v_{i}^{r}$ around $R$ is at most $\frac{k}{2}$. This path in $G^{\prime}$ forms a noncontractible nonseparating noose in $G$ (since if two cycles in a surface cross in exactly one point, then both are noncontractible).

The length of this noose in $G$ is at most $\frac{n+k}{k}-1+\frac{k}{2}$ (since $v_{i}^{\ell}$ and $v_{i}^{r}$ both appeared in the path). Hence $\frac{n+k}{k}-1+\frac{k}{2} \geq k$ by the minimality of $|V(C)|$. Thus $k \leq \sqrt{2 n}$. Therefore $G^{\prime \prime}$ has at most $n+\sqrt{2 n}+\left(\begin{array}{c}\sqrt{2 n} \\ 2\end{array}\right) \leq 3 n$ vertices.

Proof of Theorem 12. We proceed by induction on $\gamma$. If $\gamma=0$ then $G$ is planar, and $G$ itself is a planar decomposition of width $1=2^{0}$ and order $n=3^{0} n$. Otherwise, by Lemma 13, $G$ has a decomposition $D$ of width 2 , genus $\gamma-1$, and order $3 n$. By induction, $D$ has a planar decomposition of width $2^{\gamma-1}$ and order $3^{\gamma-1}(3 n)=3^{\gamma} n$. By Lemma 6 with $p=k=2$, and $\ell=2^{\gamma-1}, G$ has a planar decomposition of width $2 \cdot 2^{\gamma-1}=2^{\gamma}$ and order $3^{\gamma} n$.

Theorem 12 and Lemma 10 imply that every graph $G$ with genus $\gamma$ has crossing number $\operatorname{cr}(G) \leq 12^{\gamma} \Delta(G)^{2}|G|$, which for fixed $\gamma$, is weaker than the bound of Pach and Tóth [11] in (2). The advantage of our approach is that it generalises for graphs with an arbitrary excluded minor.

\section{$6 \quad H$-Minor-Free Graphs}

For integers $h \geq 1$ and $\gamma \geq 0$, Robertson and Seymour [13] defined a graph $G$ to be $h$-almost embeddable in $\mathbb{S}_{\gamma}$ if $G$ has a set $X$ of at most $h$ vertices such that $G \backslash X$ can be written as $G_{0} \cup G_{1} \cup \cdots \cup G_{h}$ such that:

- $G_{0}$ has an embedding in $\mathbb{S}_{\gamma}$,

- the graphs $G_{1}, G_{2}, \ldots, G_{h}$ (called vortices) are pairwise disjoint,

- there are faces $F_{1}, F_{2}, \ldots, F_{h}$ of the embedding of $G_{0}$ in $\mathbb{S}_{\gamma}$, such that each $F_{i}=V\left(G_{0}\right) \cap V\left(G_{i}\right)$,

- if $F_{i}=\left(u_{i, 1}, u_{i, 2}, \ldots, u_{i,\left|F_{i}\right|}\right)$ in clockwise order about the face, then $G_{i}$ has a strong $\left|F_{i}\right|$-cycle decomposition $Q_{i}$ of width $h$, such that each vertex $u_{i, j}$ is in the $j$-th bag of $Q_{i}$.

The following 'characterisation' of $H$-minor-free graphs is a deep theorem by Robertson and Seymour [13].

Theorem 14 ([13]). For every graph $H$ there is a positive integer $h=h(H)$, such that every $H$-minor-free graph $G$ can be obtained by $(\leq h)$-sums of graphs that are h-almost embeddable in some surface in which $H$ cannot be embedded.

Lemma 15. Every graph $G$ that is h-almost embeddable in $\mathbb{S}_{\gamma}$ has a planar decomposition of width $h\left(2^{\gamma}+1\right)$ and order $3^{\gamma}|G|$. 
Proof. By Theorem [12, $G_{0}$ has a planar decomposition $D$ of width at most $2^{\gamma}$ and order $3^{\gamma}\left|G_{0}\right| \leq 3^{\gamma}|G|$. We can assume that $D$ is connected. For each vortex $G_{i}$, add each vertex in the $j$-th bag of $Q_{i}$ to each bag of $D$ that contains $u_{i, j}$. The bags of $D$ now contain at most $2^{\gamma} h$ vertices. Now add $X$ to every bag. The bags of $D$ now contain at most $\left(2^{\gamma}+1\right) h$ vertices. For each vertex $v$ that is not in a vortex, $D(v)$ is unchanged by the addition of the vortices, and is thus connected. For each vertex $v$ in a vortex $G_{i}, D(v)$ is the subgraph of $D$ induced by the bags (in the decomposition of $G_{0}$ ) that contain $u_{i, j}$, where $v$ is in the $j$-th bag of $Q_{i}$. Now $Q_{i}(v)$ is a connected subgraph of the cycle $Q_{i}$, and for each vertex $u_{i, j}$, the subgraphs $G_{0}\left(u_{i, j}\right)$ and $G_{0}\left(u_{i, j+1}\right)$ touch. Thus $D(v)$ is connected. (This argument is similar to that used in Lemma 6.) $D(v)$ is connected for each vertex $v \in X$ since $D$ itself is connected.

Lemma 16. For all integers $h \geq 1$ and $\gamma \geq 0$ there is a constant $d=d(h, \gamma)$, such that every graph $G$ that is h-almost embeddable in $\mathbb{S}_{\gamma}$ is d-degenerate.

Proof. If $G$ is $h$-almost embeddable in $\mathbb{S}_{\gamma}$ then every subgraph of $G$ is $h$-almost embeddable in $\mathbb{S}_{\gamma}$. Thus it suffices to prove that if $G$ has $n$ vertices and $m$ edges, then its average degree $\frac{2 m}{n} \leq d$. Say each $G_{i}$ has $m_{i}$ edges. $G$ has at most $h n$ edges incident to $X$. Thus $m \leq h n+\sum_{i=0}^{h} m_{i}$. Now $m_{0}<(\sqrt{3 \gamma}+3) n$. Both endpoints of an edge of a vortex $G_{i}$ is in some bag of $Q_{i}$. Thus $m_{i} \leq\left(\begin{array}{c}h \\ 2\end{array}\right)\left|F_{i}\right|$. Since $G_{1}, G_{2}, \ldots, G_{h}$ are pairwise disjoint, $\sum_{i=1}^{h} m_{i} \leq\left(\begin{array}{c}h \\ 2\end{array}\right) n$. Thus $m<(h+$ $\left.\sqrt{3 \gamma}+3+\left(\begin{array}{c}h \\ 2\end{array}\right)\right) n$. Taking $d=h(h+1)+2 \sqrt{3 \gamma}+6$ we are done.

Lemmas [8, 15] and [6] imply:

Corollary 17. For all integers $h \geq 1$ and $\gamma \geq 0$ there is a constant $k=$ $k(h, \gamma) \geq \gamma$, such that every graph $G$ that is h-almost embeddable in $\mathbb{S}_{\gamma}$ has a planar $\omega$-decomposition of width $k$ and order $3^{\gamma}|G|$.

Now we bring in $(\leq h)$-sums.

Lemma 18. For all integers $h \geq 1$ and $\gamma \geq 0$, every graph $G$ that can be obtained by $(\leq h)$-sums of graphs that are $h$-almost embeddable in $\mathbb{S}_{\gamma}$ has a planar $\omega$-decomposition of width $k$ and order $\max \left\{1,3^{\gamma}(h+1)(|G|-h)\right\}$, where $k=k(h, \gamma)$ from Corollary 17 .

Proof. If $|G| \leq h$ then the decomposition of $G$ with all its vertices in a single bag satisfies the claim (since $k \geq h$ ). Now assume that $|G| \geq h+1$. If $G$ is $h$-almost embeddable in $\mathbb{S}_{\gamma}$, then by Corollary [17, $G$ has a planar $\omega$-decomposition of width $k$ and order $3^{\gamma}|G| \leq 3^{\gamma}(h+1)(|G|-h)$. Otherwise, $G$ is a $(\leq h)$-sum of graphs $G_{1}$ and $G_{2}$, each of which, by induction, has a planar $\omega$-decomposition of width $k$ and order $\max \left\{1,3^{\gamma}(h+1)\left(\left|G_{i}\right|-h\right)\right\}$. By Lemma 9, $G$ has a planar $\omega$-decomposition $D$ of width $k$ and order $|D|=\max \left\{1,3^{\gamma}(h+1)\left(\left|G_{1}\right|-h\right)\right\}+$ $\max \left\{1,3^{\gamma}(h+1)\left(\left|G_{2}\right|-h\right)\right\}$. Without loss of generality, $\left|G_{1}\right| \leq\left|G_{2}\right|$. If $\left|G_{2}\right| \leq h$ then $|D|=2 \leq 3^{\gamma}(h+1)(|G|-h)$. If $\left|G_{1}\right| \leq h$ and $\left|G_{2}\right| \geq h+1$, then $|D|=$ $1+3^{\gamma}(h+1)\left(\left|G_{2}\right|-h\right) \leq 3^{\gamma}(h+1)(|G|-h)$. Otherwise, both $\left|G_{1}\right| \geq h+1$ and $\left|G_{2}\right| \geq h+1$. Thus $|D| \leq 3^{\gamma}(h+1)\left(\left|G_{1}\right|+\left|G_{2}\right|-2 h\right) \leq 3^{\gamma}(h+1)(|G|-h)$. 
Proof of Theorem 3. Let $h=h(H)$ from Theorem 14. Let $\mathbb{S}_{\gamma}$ be the surface in Theorem 14 in which $H$ cannot be embedded. By Theorem 14, $G$ can be obtained by $(\leq h)$-sums of graphs that are $h$-almost embeddable in $\mathbb{S}_{\gamma}$. By Lemma 18 , $G$ has a planar $\omega$-decomposition of width $k$ and order $3^{\gamma}(h+1)|G|$, where $k=$ $k(h, \gamma)$ from Corollary 17. By Lemma 5, $G$ has a planar $\omega$-decomposition of width $k^{\prime}$ and order $|G|$, for some $k^{\prime}$ only depending on $k, \gamma$ and $h$.

Observe that Lemma 10 and Theorem 3 prove Theorem 1 .

\section{Complementary Results}

A graph drawing is rectilinear (or geometric) if each edge is represented by a straight line-segment. The rectilinear crossing number of a graph $G$, denoted by $\overline{\mathrm{cr}}(G)$, is the minimum number of crossings in a rectilinear drawing of $G$; see [2, 14]. A rectilinear drawing is convex if the vertices are positioned on a circle. The convex (or outerplanar) crossing number of a graph $G$, denoted by $\operatorname{cr}^{\star}(G)$, is the minimum number of crossings in a convex drawing of $G$; see [4, 15]. Obviously $\operatorname{cr}(G) \leq \overline{\mathrm{cr}}(G) \leq \mathrm{cr}^{\star}(G)$ for every graph $G$. Linear rectilinear and linear convex crossing numbers are defined in an analogous way to linear crossing number.

It is unknown whether an analogue of Theorem 1 holds for rectilinear crossing number 6 . On the other hand, we prove such a result for $K_{3,3}$-minor-free graphs.

Theorem 19 ([17]). Every $K_{3,3}$-minor-free graph $G$ has a rectilinear drawing in which each edge crosses at most $2 \Delta(G)$ other edges. Hence $\overline{\mathrm{cr}}(G) \leq$ $\Delta(G)\|G\| \leq \Delta(G)(3|G|-5)$.

An analogue of Theorem 1 for convex crossing number does not hold, even for planar graphs, since Shahrokhi et al. [15] proved that the $n \times n$ planar grid $G_{n}$ (which has maximum degree 4) has convex crossing number $\Omega\left(\left|G_{n}\right| \log \left|G_{n}\right|\right.$ ). Now, $G_{n}$ has tree-width $n$. In the following sense, we prove that large tree-width necessarily forces up the convex crossing number.

Theorem 20 ([17]). Every graph $G$ with degree at most $\Delta$ and tree-width at most $k$ has a convex drawing in which each edge crosses $\mathcal{O}\left(k \Delta^{2}\right)$ other edges. Hence $\operatorname{cr}^{\star}(G) \leq \mathcal{O}\left(k \Delta^{2}\|G\|\right) \leq \mathcal{O}\left(k^{2} \Delta^{2}|G|\right)$. Conversely, suppose that a graph $G$ has a convex drawing such that whenever two edges $e$ and $f$ cross, $e$ or $f$ crosses at most $\ell$ edges. Then $G$ has tree-width at most $3 \ell+11$.

In particular, graphs of bounded degree and bounded tree-width have linear convex crossing number. Again, the assumption of bounded degree is necessary since $K_{3, n}$ has tree-width 3 and crossing number $\Omega\left(n^{2}\right)$.

\footnotetext{
$\overline{6}$ The crossing number and rectilinear crossing number are not related in general. In particular, for every integer $k \geq 4$, Bienstock and Dean [2] constructed a graph $G_{k}$ with crossing number 4 and rectilinear crossing number $k$. It is easily seen that $G_{k}$ has no $K_{14}$-minor. However, the maximum degree of $G_{k}$ increases with $k$. Thus $G_{k}$ is not a counterexample to an analogue of Theorem 1 for rectilinear crossing number.
} 
Acknowledgements. Thanks to János Pach and Géza Tóth for explaining their proofs in reference [1].

\section{References}

[1] Therese Biedl, Erik D. Demaine, Christian A. Duncan, Rudolf FleisCher, And Stephen G. Kobourov. Tight bounds on maximal and maximum matchings. Discrete Math., 285(1-3):7-15, 2004.

[2] Daniel Bienstock and Nathaniel Dean. Bounds for rectilinear crossing numbers. J. Graph Theory, 17(3):333-348, 1993.

[3] Drago Bokal, Gašper Fijavž, and Bojan Mohar. The minor crossing number. SIAM J. Discrete Math., 20(2):344-356, 2006.

[4] Éva Czabarka, Ondrej SÝkora, László A. SzÉkely, and Imrich Vrt'o. Outerplanar crossing numbers, the circular arrangement problem and isoperimetric functions. Electron. J. Combin., 11(1):R81, 2004.

[5] Reinhard Diestel. Graph theory, vol. 173 of Graduate Texts in Mathematics. Springer, 2nd edn., 2000.

[6] Reinhard Diestel and Daniela Kühn. Graph minor hierarchies. Discrete Appl. Math., 145(2):167-182, 2005.

[7] Hristo N. DJidjev And ImRich VRŤo. Planar crossing numbers of genus $g$ graphs. In Proc. 33rd International Colloquium on Automata, Languages and Programming (ICALP '06), vol. 4051 of Lecture Notes in Comput. Sci., pp. 419 430. Springer, 2006.

[8] Enrique Garcia-Moreno and Gelasio Salazar. Bounding the crossing number of a graph in terms of the crossing number of a minor with small maximum degree. J. Graph Theory, 36(3):168-173, 2001.

[9] Jesús Leaños and Gelasio Salazar. On the additivity of crossing numbers of graphs, 2006.

http://www.ifisica.uaslp.mx/〜gsalazar/RESEARCH/additivity.pdf.

[10] Bojan Mohar and Carsten Thomassen. Graphs on surfaces. Johns Hopkins University Press, Baltimore, U.S.A., 2001.

[11] JÁnos PACH ANd GÉzA Tóth. Crossing number of toroidal graphs. Proc. 13th International Symp. on Graph Drawing (GD '05), vol. 3843 of Lecture Notes in Comput. Sci., pp. 334-342. Springer, 2006.

[12] R. Bruce Richter and Jozef Śríñ . The crossing number of $K_{3, n}$ in a surface. J. Graph Theory, 21(1):51-54, 1996.

[13] Neil Robertson and Paul D. Seymour. Graph minors. XVI. Excluding a non-planar graph. J. Combin. Theory Ser. B, 89(1):43-76, 2003.

[14] Edward R. Scheinerman and Herbert S. Wilf. The rectilinear crossing number of a complete graph and Sylvester's "four point problem" of geometric probability. Amer. Math. Monthly, 101(10):939-943, 1994.

[15] Farhad Shahrokhi, Ondrej SÝkora, Laszlo A. Székely, and Imrich VRT'o. The gap between crossing numbers and convex crossing numbers. Towards a Theory of Geometric Graphs, vol. 342 of Contemporary Mathematics, pp. 249258. Amer. Math. Soc., 2004.

[16] LÁszló A. SzÉKELY. A successful concept for measuring non-planarity of graphs: the crossing number. Discrete Math., 276(1-3):331-352, 2004.

[17] David R. Wood and Jan Arne Telle. Planar decompositions and the crossing number of graphs with an excluded minor. 2006.

http://www .arxiv.org/math/0604467. 